\section{A comparative investigation into effects of L1 lexicalization and cultural loadedness on incidental vocabulary acquisition and retention}

\author{
Heidari-Shahreza, Mohammad Ali \\ University of Isfahan, Iran (maheidari.sh@gmail.com) \\ Moinzadeh, Ahmad \\ University of Isfahan, Iran (moin@fgn.ui.ac.ir) \\ Barati, Hossein \\ University of Isfahan, Iran (h.barati@gmail.com)
}

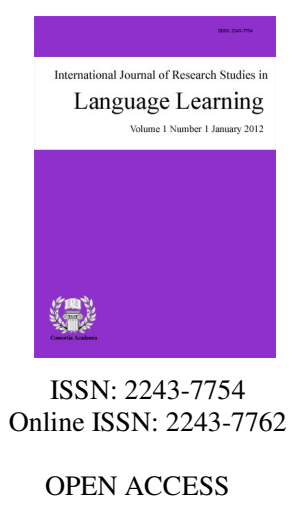

\title{
Abstract
}

Of primary importance to this study was to compare the incidental acquisition and retention of non-lexicalized (NL) and culturally-loaded (CL) words by 90 Persian-speaking EFL learners. An NL word was defined as an English word which despite bearing the same meaning(s) could not be translated with one single word in the learners' L1 (i.e. Persian). CL words also referred to the lexical items which despite the same primary meaning(s) in both languages (i.e. Persian and English), bore significantly-different cultural connotations. The findings revealed that both NL and CL words were significantly different in the semantic aspects of vocabulary knowledge such as meaning and associations. Nevertheless, NL words were more sensitive to the number of repetition. It seems both groups of words may cause extra difficulty for EFL learners. Thus, the researchers recommend that language teachers have a special focus on such troublesome words through an intensive, systematic recycling program.

Keywords: non-lexicalized words; culturally-loaded words; vocabulary knowledge; Persian language; EFL learners 


\section{A comparative investigation into effects of $L 1$ lexicalization and cultural loadedness on incidental vocabulary acquisition and retention}

\section{Introduction}

It is generally agreed that the ultimate aim of second language (L2) acquisition in EFL contexts is for the learners to communicate effectively in that language (Ghafar-Samar \& Dehqan, 2013). In this regard, vocabulary knowledge (or lexical competence) is widely recognized by many vocabulary acquisition researchers as a fundamental component of language acquisition and communicative competence (Hairrell, Rupley, \& Simmons, 2011; Nation, 2013; Ang, 2014). Knowledge of vocabulary is also closely associated with the four traditional languages skills of reading, writing, listening and speaking (Brown, Waring, \& Donkaewbua, 2008; Crossley, Salsbury, \& McNamara, 2011; Mehrpour \& Rahimi, 2010; Karami \& Salahshoor, 2014). Therefore, in the realm of second language acquisition (SLA), research on the acquisition and retention of various aspects of vocabulary knowledge are worthwhile lines of investigation (Hairrell et al., 2011; Nation, 2013; Webb, 2007). Within the perspective of L2 vocabulary acquisition, scholars have defined knowledge of vocabulary in a number of ways, most often emphasizing the multidimensional nature of this knowledge (Kieffer \& lesaux, 2012; Laufer, Elder, Hill, \& Congdon, 2004; Qian \& Schedl, 2004). In this regard, distinctions are made with respect to vocabulary 'breadth' or the number of words learners know (even partially) and vocabulary 'depth' or how well learners are familiar with the different uses and connotations of L2 words in authentic contexts (Hellman, 2011; Marzban \& Hadipour, 2012; Webb, 2013).

Likewise, Nation (2001), views vocabulary knowledge as incorporating subknowledges of form, meaning and use both at recognition (receptive knowledge) and production level, productive knowledge (see also Webb, 2009). In short, studies in L2 vocabulary acquisition have explored various aspects of vocabulary knowledge such as spelling, syntactic functions, meaning and associations from both an explicit (intentional) and implicit (or incidental) point of view (Brent \& Siskind, 2001; Chen \& Truscott, 2010; Heidari-Shahreza \& Tavakoli, 2012; Pulido, 2004; Kim, 2011; Min, 2008, to name a few). While scholars differ in the extent they favor one approach over the other (see Choo, Lin, \& Pandian, 2012; Hairrell et al., 2011 for a review), incidental vocabulary acquisition (i.e. learning new words in the context of reading) is generally assumed to be a notable means of vocabulary development particularly after the first few thousands words of L2 vocabulary, at intermediate and more advanced levels of language proficiency (Pellicer-Sanchez \& Schmitt, 2010; Rott, 2013). Despite three decades of research on incidental vocabulary acquisition and retention, however, two important issues have not received much attention in the literature (Khatib \& Nourzadeh, 2012 for a review on incidental vocabulary acquisition).

\subsection{L1 lexicalization}

The first issue is concerned with L1 lexicalization. 'Lexicalization' in general refers to the way a language molds a given concept into word(s) (Chen \& Truscott, 2010). Simply put, an identical concept may be represented by a different number of words across languages (Paribakht, 2005). Therefore, while in English, for instance, the concept of 'bringing down one's foot on something on the ground' is expressed by the single word 'stamp', it needs to be expressed (or translated) into Persian, in several words as ' جيزى را لكد كردن جئ '. Thus, an English word like 'stamp' is regarded as non-lexicalized (NL) with respect to Persian. 'Laugh', however, is conveniently replaced with خنديدن ' (xandidan) in Persian. Therefore, it is an example of a lexicalized (L) word in English. It is of primary importance to see how the acquisition and retention of NL words might be different from other L2 vocabulary. 
The effects of L1 lexicalization and cultural loadedness on incidental vocabulary acquisition and retention

\subsection{Cultural loadedness}

A second neglected issue in the realm of vocabulary acquisition and retention is 'cultural loadedness'. It refers to significantly excess cultural connotations that some L2 words might convey with respect to learners' L1, most often associated with negative feelings, communicative inappropriateness or religious do's and don'ts (Liu \& Zhong, 1999). Comparing English and Persian again, the English word 'girlfriend', for instance, is lexically and semantically translatable in Persian. Yet, within the Islamic context where most Persian speakers come from, it arouses strong feelings of inappropriateness, resulting from Islamic constraints on acceptable male/female relationships. Therefore, this word is regarded as culturally-loaded as far as Persian or culturally similar languages are concerned. In this study, culturally-loaded (CL) words are placed against culturally-neural (CN) words which represent the larger group of L2 vocabulary that imply more or less the same cultural connotations across languages (here English and Persian). In addition, it is worth noting that identifying NL and CL words (as well as their neutral counterparts) and also deciding upon which words to include and which not required great care and caution. This is hopefully achieved in this study through the researchers' joint endeavor with 40 volunteers to select 20 appropriate words out of 1500 suggested words over a period of six months (see section 3.2 on 'target words' for further information).

\section{Literature review}

\subsection{Research on L1 lexicalization}

The issue of L1 lexicalization has received little attention until recently. Paribakht (2005) is among the first to address this research topic. She investigated the inferencing behavior of 20 Persian-speaking EFL learners in comprehending six English texts in which a total of 50 target words (both L and NL) were embedded. The findings indicated that the participants were much less successful in comprehending NL words, even though they relied on the same knowledge sources for the both sets of target words. Paribakht concluded that L1 lexicalization might significantly contribute to the overall success of EFL learners in L2 reading comprehension and vocabulary development. However, her study did not explore the acquisition of NL words since it was focused on only lexical inferencing.

Chen and Truscott (2010) also explored the impact of L1 lexicalization on incidental vocabulary acquisition and two-week retention of 10 target words by 72 adult EFL learners. Among other things, they found that NL words could be far more difficult for the learners. Their findings, however, were affected by the position and saliency of the target words in the composed reading passages used in the study.

In a similar study, based on Chen and Truscott's study (2010), Heidari-Shahreza and Tavakoli (2012) examined the possible effects of L1 lexicalization and the number of repetition on successful vocabulary acquisition and retention of 10 target words. They had their 90 EFL learners read seven reading passages through which they encountered the target words for one, three or seven times. As for L1 lexicalization, the results revealed that there were significant differences between L and NL words in the semantic aspects of vocabulary knowledge such as meaning and association, resulting in learners' difficulty in dealing with NL English words. Nevertheless, this study was also limited in that the cultural connotations of the target words were not fully controlled for.

Golaghaei and Sadighi (2013) also explored the impact of providing (non)-interventionist treatments for both sets of L and NL target words on the acquisition of vocabulary knowledge by 74 adult EFL learners. The findings demonstrated that the learners had greater familiarity with L words at pre-testing, and they were more successful in learning them at post-testing. Moreover, there was no significant difference between L and NL words in the non-interventionist group. Convincing conclusions, however, cannot be drawn from this study, as the researchers were mainly concerned with explicit instruction (i.e. interventionist treatment) of the target words rather than their incidental acquisition. 


\subsection{Research on cultural loadedness}

While the importance of CL words has been recognized for a long time, to date, research on cultural loadedness has been scarce (Zhao, 2004). Moreover, this scant body of research mainly centers on comparisons between EFL/ESL learners and native speakers in terms of their perception and use of CL words, not their acquisition and retention of these words in any depth. Qi (1992), for instance, explored the effect of an L2 prior cultural awareness on lexical meaning-making of CL words. The participants were three Chinese ESL learners and one native speaker. The findings revealed that in spite of an overlapping conceptual relationship in the core meaning of CL words between native and non-native participants, there were significant differences in how the two groups perceived connotations of these words.

Liu and Zhong (1999) also asked 125 EFL learners and 61 native speakers of English to rate the appropriateness of six CL and four CN words. The findings revealed that even advanced EFL participants had difficulty in understanding CL words. The authors suggested that that more attention should be paid to such words in language learning and teaching. In a similar study, Kupelian (2001) compared 108 Korean EFL learners' understanding of $11 \mathrm{CL}$ and four CN English words with that of 54 native English speakers. He also examined the effect of studying English in an English-speaking country on the learners' cultural understanding with those EFL participants who had studied English in such a country for more than four months. The findings, in general, indicated that all EFL learners had inadequate awareness of English CL words, as concluded by Liu and Zhong (1999).

In a somewhat related study, Zhao (2004) explored the effect of enhanced cultural awareness on the performance of 40 advanced Chinese students in evaluating the appropriate usage of $20 \mathrm{CL}$ words. The findings, in general, indicated that increasing the participants' awareness of the target words' cultural connotations could significantly affect their correct judgment of CL words in English texts.

\section{Method}

As the scarcity of studies on L1 Lexicalization and cultural loadedness implies, further research on the effects of these two factors is needed. Moreover, to the best of the researchers' knowledge, this is the first study to compare and contrast these factors together. Therefore, the present study, through a quasi-experimental design, explores and compares the possible effects of L1 lexicalization and cultural loadedness incidental vocabulary acquisition of 90 Persian-speaking EFL learners. Furthermore, retention (within a three-week span) is taken into account by a delayed posttest. This study is part of a larger project investigating number of repetition and exposure frequency, L1 lexicalization and L1-L2 lexical mismatch, and cultural loadedness (to be reported).

Based on the purpose of this study, four research questions were addressed:

1. Do EFL learners have difficulty in learning target words which are not lexicalized in their L1? Which aspects of vocabulary knowledge are more involved?

2. Do EFL learners have difficulty in learning target words that are culturally-loaded in comparison with their L1? Which aspects of vocabulary knowledge are more involved?

3. To what extent the observed effects of L1 lexicalization and cultural loadedness, if any, are retained after a three-week delay?

4. How do the acquisition and retention of culturally-loaded words differ from non-lexicalized words?

\subsection{Participants}

The participants of the study were first- and second-year students of an Iranian university. First, based on the scores obtained from Oxford Placement Test, 111 students were determined as intermediate. Then, they sat for 
The effects of L1 lexicalization and cultural loadedness on incidental vocabulary acquisition and retention

the Vocabulary Levels Test (Nation 1990), a widely-used size test that is an appropriate measurement instrument to assess learners' vocabulary knowledge before an experiment (Laufer \& Goldstein, 2004; N. Schmitt, D. Schmitt, \& Clapham, 2001).

All participants scored 25 or more (out of 30) on 2000 level of the Vocabulary Level Test, with an average score of 28.2. Since the socio-cultural background of the participants could affected the internal validity of this research, they also answered a socio-cultural survey in which there were questions about the participants' first (or dominant) language, their affinity with other cultures and amount of exposure they had to English. A few participants were excluded from the study because of their significantly different score on the Levels test or their different socio-cultural background. For instance, while one of them spoke Persian fluently, it was revealed that he spoke mainly Turkish outside academic settings. Finally, 90 participants were discerned to be appropriate for this study that were then, divided into three groups of 30, based on the number of encounters to target words (i.e. E1, E3 and E7). The participants were also informed that their participation in this study was voluntary and that leaving the study would not result in any penalty or affect their relation with their instructors at the university at issue

\subsection{Materials and Instruments}

The following material and instruments were employed to carry out the study:

Target words (TWs) - There were 20 target words (TWs, hereafter) which were evenly divided into four categories: Lexicalized (L); Non-lexicalized (NL); Culturally-loaded (CL); Culturally-neutral (CN)

There were eight verbs, eight nouns and four adjectives (see Table 1). To choose the TWs, following the same procedure as Paribakht (2005) did, 40 university students who were native speakers of Persian and fluent in English volunteered to prepare a list of words from their English readings over a period of 6 months that, based on their intuition, could fall into the categories of the TWs (i.e. L, NL, CL or CN). Each of them was asked to possibly find 40 words in different syntactic functions (10 words for each category, i.e. L, NL, CL and CN with four verbs, three nouns, two adjectives and one adverb). They also checked their initial guess about the suitability of a word against well-established English as well as Persian dictionaries and native speakers. In addition, the researchers asked the assistants to provide at least one example of the authentic use of each TW.

Before and over these 6 months, the researchers held several meetings with the voluntary assistants to answer any questions they might have. During these meetings, examples of appropriate TWs were discussed and the essential characteristics of possible TWs were explained. In the end, more than 1500 words were collected. The researchers themselves, once again, checked these words against several English-Persian dictionaries and consulted several Persian as well as English native speakers and linguists. A few words were discarded. They, then, chose the final 20 TWs based on the difficulty level, conceptual familiarity, word frequency and of course, the characteristics of L, NL, CL and CN words. Using a checklist, the researchers made sure that the final TWs were unknown to the participants prior to the experiment.

\section{Table 1}

Selected target words

\begin{tabular}{llll}
\hline Lexicalized & Non-lexicalized & Culturally-loaded & Culturally-neutral \\
\hline explain $(\mathrm{v})$ & elope $(\mathrm{v})$ & gamble $(\mathrm{v})$ & hang $(\mathrm{v})$ \\
flee $(\mathrm{v})$ & giggle $(\mathrm{v})$ & date $(\mathrm{v})$ & smuggle $(\mathrm{v})$ \\
annoyance $(\mathrm{n})$ & lounge $(\mathrm{n})$ & pet $(\mathrm{n})$ & junction $(\mathrm{n})$ \\
masterpiece $(\mathrm{n})$ & brunch $(\mathrm{n})$ & undertaker $(\mathrm{n})$ & wholesale $(\mathrm{n})$ \\
stubborn $(\operatorname{adj})$ & smoggy $(\operatorname{adj})$ & abstinent $(\operatorname{adj})$ & retired $(\operatorname{adj})$ \\
\hline
\end{tabular}

Reading passages - The researchers working with two native English teachers wrote seven main reading passages (M) which each contained all $20 \mathrm{TWs}$ once. Moreover, to have the participants read the same number 
of reading passages despite the different number of encounters to TWs, six distracter passages (D) were also taken from a reading textbook at intermediate level (Kirn \& Hartmann, 2002). Therefore, on the whole, there were 13 reading passages of almost the same length ( 250 words) and difficulty level but only seven of them (i.e. Main passages) contained the TWs (see Table 2).

Table 2

Distribution of reading passages

\begin{tabular}{|c|c|c|}
\hline Group & Distribution of main and distracter passages & Exposure \\
\hline E1 & $\begin{array}{lllllll}\mathrm{D}_{1} & \mathrm{D}_{2} & \mathrm{D}_{3} & \mathrm{D}_{4} & \mathrm{D}_{5} & \mathrm{D}_{6} & \mathrm{M}_{7}\end{array}$ & 1 \\
\hline E3 & $\begin{array}{lllllll}M_{1} & D_{2} & D_{3} & M_{4} & D_{5} & D_{6} & M_{7}\end{array}$ & 3 \\
\hline E7 & $\begin{array}{lllllll}M_{1} & M_{2} & M_{3} & M_{4} & M_{5} & M_{6} & M_{7}\end{array}$ & 7 \\
\hline
\end{tabular}

Vocabulary post-test - The vocabulary posttest used in this study was based on Chen and Truscott's (2010) and Heidari-Shahreza and Tavakoli's (2012) test of vocabulary knowledge. As shown in Table 3, this test addressed various receptive and productive facets of vocabulary knowledge using different forms of measurement such as L1 translation, multiple-choice test, etc. The test was implemented in practice as follows:

\section{Table3}

Types of vocabulary knowledge \& vocabulary post-test subtests

\begin{tabular}{lll}
\hline Order & \multicolumn{1}{c}{ Knowledge measured } & \multicolumn{1}{c}{ Test type } \\
\hline 1 & Productive knowledge of orthographic form (PO) & Dictation \\
2 & Receptive knowledge of orthographic form (RO) & Multiple choice \\
3 & Receptive knowledge of meaning and form (RMF) & Translation (L2-L1) \\
4 & Productive knowledge of parts of speech (PP) & Sentence construction \\
5 & Productive knowledge of associations (PA) & Pragmatic association \\
6 & Receptive knowledge of parts of speech (RP) & Multiple choice \\
7 & Receptive knowledge of associations (RA) & Multiple choice \\
\hline
\end{tabular}

Sub-test 1. Productive knowledge of orthographic form (PO): A dictation test was used to measure this aspect of vocabulary knowledge. The learners listened to each TW twice from a recording and had 20 seconds to write it on their answer sheet. To block out the effect of phonological familiarity on the participants' spelling, (Webb 2007; Chen \& Truscott, 2010), an item was marked as correct if only there was no error whatever in the spelling of the TW.

Sub-test 2. Receptive knowledge of orthographic form (RO): A four-option multiple-choice format was used to measure the receptive knowledge of orthographic form. The three distracters of each test item were misspelled forms of each TW. The following is for the TW, 'abstinent':

$\begin{array}{llll}\text { a. abstinent } & \text { b. abstinant } & \text { c. absctinent } & \text { d. abstenent }\end{array}$

Sub-test 3. Receptive knowledge of meaning and form (RMF): To test receptive knowledge of meaning and form, the participants translated the TWs into their L1 (i.e. Persian). Their translations needed to incorporate all essential semantic features of the TWs. Taking the word 'smuggle' as an example, an answer such as 'move' was considered as wrong for the semantic component [+ illegal] was not embedded.

Sub-test 4. Productive knowledge of parts of speech (PP): To assess this aspect of vocabulary knowledge, the researchers instructed the participants to use the TWs in sentences in English. In this test, the focus was on the use of TWs in the grammatical functions they had appeared in reading passages.

Sub-test 5. Productive knowledge of associations (PA): The learners, on this test, had to think of a word which was pragmatically related to the TWs. As an example, for the TW, 'giggle', an answer such as 'laugh' was appropriate. In addition, the participants could not provide syntactic associations for this test.

Sub-test 6. Receptive knowledge of parts of speech (RP): To assess the learners' receptive knowledge of 
The effects of L1 lexicalization and cultural loadedness on incidental vocabulary acquisition and retention

parts of speech, each TW appeared in three similar sentences but in different grammatical functions. The participants were expected to check the sentence containing the TW in the correct grammatical function. See the example below for the TW, 'elope' as a verb:

$>$ Alice ran elopely to Spain with her boyfriend.

$>\quad$ An elope to Spain was her boyfriend's idea.

$>\quad$ Alice eloped with his boyfriend to Spain. ${ }^{\checkmark}$

Sub-test 7. Receptive knowledge of associations (RA): On this subtest, each TW was accompanied with five words. The Participants were instructed to choose the two words which were pragmatically associated with the TW. Following Chen and Truscott (2010) and Heidari-Shahreza and Tavakoli (2012); more than one correct response was allowed to see if or not the learners had acquired all semantic features of NL and CL words. Here is the TW, 'masterpiece' with its five accompanying options.

$\mathrm{TW}=$ masterpiece

(a) work ${ }^{2}$ (b) part (c) $\operatorname{artist}^{2}$ (d) slave (e) land

It is also worth mentioning that the reliability and validity of the vocabulary posttest were checked through the process of piloting the subtests. Several response distracters were revised and the instructions were made clearer. The average reliability was calculated as 0.87 .

\subsection{Data collection}

The data collection procedure was conducted as follows:

Reading passages - Based on the design of the study, the participants were randomly assigned to one of the three experimental groups (i.e. E1, E3 or E7). All participants read the same number of reading passages. However, the number of main and distracter passages differed in each group. That is, group E1 read six distracter passages and only its seventh passage contained the TWs. The first, fourth and seventh passage for group E3 was main while the other four texts were just distracters. As for E7, all reading passages were main, containing the TWs. It should be said that the researchers sequence the distracter passages in a way that the seventh passage for all groups contained the TWs (i.e. it was a main passage). This way, there was no difference in how recently the learners had come across the TWs (see Table 2). The reading phase took about 50 minutes. That is, on average, the participants spent seven minutes to finish reading each text. This amount of time was assured to be adequate through piloting procedure.

Immediate post-test - Once the participants had finished reading the passages, the vocabulary post-test was given out. Each subtest was printed on a single page and instructions were supplied as not to go back to the previous subtests. No test duration was set. However, almost all test-takers submit their answer sheets within a reasonable time limit. Generally, the participants remained attentive until the end of the test (as far as it was apparent).

Delayed post-test - After a lapse of three weeks, again, the participants were kindly invited to participate in the research. Fortunately, all of them took part. Following the same procedure as the immediate post-test, the delayed test was administered and the answer sheets were collected for later scoring and analysis. While it is practically hard to claim, the researchers did their best to control for any significant effect from the participants' exposure to English on their performance during these three weeks by monitoring their learning tasks and the reading materials they were assumed to be involved in.

Afterwards, the administered immediate and delayed post-tests were scored systematically by the researchers and two assistants who were experienced EFL teachers with sufficient knowledge in teaching and 
testing. Before scoring the tests, several meetings were held between the researchers and their assistants to define and clarify the research scope and the scoring procedure. Therefore, all scorers followed the same blueprint in scoring the tests. The inter-rater reliability was also sufficiently high (0.85).To score the vocabulary post-test, each question item (in each subtest) was assigned half a point. Therefore, each subtest had 10 points and the overall test had 70 points. It is worth noting that in the seventh subtest (i.e., receptive knowledge of associations subtest), two correct answers were required in each question item. Thus, a quarter a point was devoted to each correct answer. This way, all question items and the subtests had the same scoring value.

\subsection{Data analysis}

To explore the effect of L1 lexicalization and cultural loadedness on incidental vocabulary acquisition, first, the scores obtained from the administration of the immediate and delayed post-tests on the three groups of participants (E1, E3 and E7) were analyzed using ANOVA and its non-parametric version Kruskal-Wallis whenever normality requirement was not met. In addition, Post hoc Tukey and least significance difference (LSD) tests were employed to specify significant effects (at $p=.05$ ). In the following section, the results based on these analyses are presented in details.

\section{Results}

In this section, the results of the study obtained in the immediate and delayed phases are reported.

\subsection{Effects of L1 lexicalization and Cultural loadedness in the immediate post-test}

As Table 4 shows the participants in all groups obtained significantly higher scores for $\mathrm{L}$ words in the Receptive Knowledge of Associations (RA) test; those in group E3, and E7 also had a significantly better performance on the Receptive Knowledge of Meaning and Form (RMF) test for L words. Also, the scores for L words were significantly higher on the Productive Knowledge of Associations (PA) test only for group E7. In all other cases, the differences between L and NL word scores did not reach statistical significance. As for cultural loadedness, following the same statistical procedure, the obtained results resembled those of L vs. NL words in the immediate post-test in that there was a significant difference between the mean scores for CL words compared with CN words for group E7 on the three subtests of Receptive Knowledge of Meaning and Form (RMF), Productive Knowledge of Associations (PA) and Receptive Knowledge of Associations (RA). However, in all other cases, there were no statistically significant differences between $\mathrm{CL}$ and $\mathrm{CN}$ words which differ from scores obtained for $\mathrm{L}$ and $\mathrm{NL}$ words where there was a significant difference between the mean scores for $\mathrm{L}$ words compared with NL words for all groups in the Receptive Knowledge of Associations (RA) test. In addition, on the Receptive Knowledge of Meaning and Form (RMF) test, the scores for L words were significantly higher not only for group E7 but also group E3.

\section{Table 4}

Comparison between $L, N L, C L$ and $C N$ words in the immediate post-test

\begin{tabular}{|c|c|c|c|c|c|c|}
\hline Group & E1 & & E3 & & E7 & \\
\hline Subtest & $\mathrm{L} \leftrightarrow \mathrm{NL}$ & $\mathrm{CL} \leftrightarrow \mathrm{CN}$ & $\mathrm{L} \leftrightarrow \mathrm{NL}$ & $\mathrm{CL} \leftrightarrow \mathrm{CN}$ & $\mathrm{L} \leftrightarrow \mathrm{NL}$ & $\mathrm{CL} \leftrightarrow \mathrm{CN}$ \\
\hline Orthography (P) & 0.243 & 0.145 & 0.465 & 0.455 & 0.402 & 0.352 \\
\hline Orthography (R) & 0.632 & 0.645 & 0.487 & 0.376 & 0.584 & 0.346 \\
\hline Meaning \& form $(\mathrm{R})$ & 0.246 & 0.431 & $0.003^{*}$ & 0.263 & $0.024 *$ & $0.045^{*}$ \\
\hline Parts of speech $(\mathrm{P})$ & 0.453 & 0.134 & 0.541 & 0.319 & 0.218 & 0.265 \\
\hline Associations (P) & 0.279 & 0.347 & 0.468 & 0.634 & $0.003^{*}$ & $0.026^{*}$ \\
\hline Parts of speech (R) & 0.734 & 0.562 & 0.354 & 0.296 & 0.386 & 0.441 \\
\hline Associations (R) & 0.254 & 0.205 & $0.023 *$ & 0.219 & $0.005^{*}$ & $0.033 *$ \\
\hline
\end{tabular}

Note. ${ }^{*}=\mathrm{p}<.05$; L: Lexicalized; NL: Non-lexicalized; CL: Culturally-loaded; CN: Culturally-neutral; P: Productive; R: Receptive 
The effects of L1 lexicalization and cultural loadedness on incidental vocabulary acquisition and retention

\subsection{Effects of L1 lexicalization and cultural loadedness in the delayed post-test}

The same statistical procedure was repeated to locate any significant difference between the mean scores for L and NL words on the delayed post-test. Unlike the immediate post-test, this time, the mean score differences between L and NL words in group E7 did not remained significant on the Productive Knowledge of Associations (PA) test. As for the other subtests, the results resembled those of the immediate posttests (see Table 5). Regarding the mean scores for CL and CN words on the delayed post-test, the same significant differences were reached as for the immediate post-test on Receptive Knowledge of Meaning and Form (RMF) and Receptive Knowledge of Associations (RA) for group E7. However, unlike the immediate post-test, the mean score differences between $\mathrm{CL}$ and $\mathrm{CN}$ words did not reach statistical significance on Productive Knowledge of Associations for group E 7. Moreover, whereas on the Receptive Knowledge of associations, the mean score differences between L and NL words for group E7 did not remain significant in the delayed post-test, the observed differences between $\mathrm{CL}$ and $\mathrm{CN}$ words again reached statistical significance which among other things may reveal different nature of CL words in comparison with NL words.

\section{Table 5}

Comparison between $L, N L, C L$ and $C N$ words in the delayed post-test

\begin{tabular}{lllllll}
\hline Group & E1 & \multicolumn{3}{c}{ E3 } & E7 \\
\hline Sub-test & $\mathrm{L} \leftrightarrow \mathrm{NL}$ & $\mathrm{CL} \leftrightarrow \mathrm{CN}$ & $\mathrm{L} \leftrightarrow \mathrm{NL}$ & $\mathrm{CL} \leftrightarrow \mathrm{CN}$ & $\mathrm{L} \leftrightarrow \mathrm{NL}$ & $\mathrm{CL} \mathrm{CN}$ \\
Orthography (P) & 0.530 & 0.234 & 0.223 & 0.359 & 0.489 & 0.390 \\
Orthography (R) & 0.557 & 0.701 & 0.499 & 0.541 & 0.798 & 0.318 \\
Meaning \& form (R) & 0.288 & 0.341 & $0.020^{*}$ & 0.357 & $0.001^{*}$ & $0.038^{*}$ \\
Parts of speech (P) & 0.587 & 0.360 & 0.876 & 0.274 & 0.323 & 0.402 \\
Associations (P) & 0.119 & 0.195 & 0.273 & 0.476 & 0.334 & 0.239 \\
Parts of speech (R) & 0.456 & 0.632 & 0.564 & 0.538 & 0.704 & 0.307 \\
Associations (R) & 0.062 & 0.481 & $0.006^{*}$ & 0.520 & $0.021^{*}$ & $0.012^{*}$ \\
\hline Note * $=$ p < 05; L: Lexicalized; NL: Non-lexicalized; CL· Culturally-loaded; CN: Culturally-neutral; P: Productive; R: Receptive
\end{tabular}

Note. ${ }^{*}=\mathrm{p}<.05$; L: Lexicalized; NL: Non-lexicalized; CL: Culturally-loaded; CN: Culturally-neutral; P: Productive; R: Receptive

\section{Discussions}

The present study compared and contrasted the acquisition and retention of lexicalized (L) vs. non-lexicalized (NL) words, on the one hand and culturally-loaded (CL) vs. culturally-neutral (CN) words on the other hand. The findings generally indicated that the main difference between NL as well as CL words and their counterparts (i.e. L and CN words, respectively) lies in the semantic aspects of vocabulary knowledge. That is to say, there were significant differences between scores obtained on the Receptive Knowledge of Meaning and Form (RMF) and Receptive Knowledge of Associations (RA) both immediately and after three weeks for group E7. In this regard, while the mean score differences for the Productive Knowledge of Associations (PA) subtest were significant in the immediate post-tests (for both NL vs. L and CL vs. CN words), it did not reach statistical significance again in the delayed test. The reason might be that in PA test, the participants needed to provide an L2 word that was pragmatically associated to the target words (TWs). Nevertheless, in RMF and RA tests, they had to merely identify the correct options. Therefore, while the former subtest (i.e. PA) required vocabulary knowledge competence at the level of production, these latter tests only entailed recognition. In other words, as Webb (2007) points out, receptive measures generally respond better to even small gains of knowledge.

Furthermore, although the results generally suggest that the acquisition and retention of CL words follow the same pattern as NL ones, a significant difference between the two sets of the TWs was seen in the subtest of Receptive Knowledge of Associations (RA) in that while significant differences were observed and retained (after three weeks) between L and NL words even after three encounters (E3), the mean score differences between CL and CN words reached statistical significance only after seven encounters (E7). Despite the need for further research in this regard, it might be the case that whereas there is only lexical (or formal) mismatch between $\mathrm{L}$ and NL words, in CL words (as their name suggests), there also exists a semantic mismatch (or even gap) to be compensated for as learners encounter such words in different contexts, absorbing their semantic 
Heidari-Shahreza, M. A., Moinzadeh, A., \& Barati, H.

(cultural) nuances (see Corrigan, 2007; Erten \& Tekin, 2008; Jiang, 2004 for some related studies).

The Receptive Knowledge of Meaning and Form is another aspect of vocabulary knowledge in which CL words appeared to be different from NL words. As for RA subtest, the mean score differences between L and NL words were calculated as significant after three encounters both immediately and after a three-week delay. But, it required at least seven encounters for CL words. While not sure, the researchers think that this difference also originates from the somewhat different nature of CL words and the RMF test. That is to say, in this test, the participants had to translate the TWs into their L1, covering all essential semantic components of each TW. However, this process could be deterred by the fact that the initial form-meaning linkage of an L2 word is usually mediated by an L1 translation in which the L2 form is attached to an already existing L1 meaning (Barcroft, 2002; Hall, 2002; Hamada, 2009; Larrañaga, Treffers-Daller, Tidball, \& Ortega, 2012). Since in the case of CL words, these L1 meanings lack (or excessively are loaded with) some (essential) semantic components of the TWs, the participants are probably less successful in providing appropriate L1 words, the ones covering all necessary semantic features of the TWs until these learners are either explicitly instructed or incidentally acquired.

\section{Conclusions}

Of primary importance to this study was to compare the incidental acquisition and retention of NL and CL words. An NL word was operationally defined as an English word which despite bearing the same meaning could not be translated (or replaced) with one single word in the learners' L1 (i.e. Persian). CL words also referred to the lexical items which in spite of the same primary meaning(s) in both languages, bore significantly-different cultural connotations. These two sets of words were juxtaposed with their neutral counterparts that actually represented the huge bulk of English vocabulary to discern how they would differ. The findings generally suggested that both NL and CL words were different from the other two sets of the TWs in the semantic aspects of vocabulary knowledge such as meaning and associations. Nevertheless, NL words appeared to be more sensitive to the number of encounters than the CL ones as significant differences between L and NL words were identified in the Receptive Knowledge of Associations (RA) and Receptive Knowledge of Meaning and Form (RMF) even after three encounters but it took seven encounters for the other group of words at issue.

In sum, it seems both NL and CL words may cause extra difficulty for EFL learners. Therefore, it is recommended that English teachers have a special focus on these words. The primary concern of teachers should be to ensure that EFL learners are able to appreciate and acquire the semantic (and lexical) features NL and CL words have, particularly those not existent in their L1. While it is easier said than done, encouraging the learners to read extensively authentic English texts might help to this end (See Day \& Bamford, 2002; Pellicer-Sanchez \& Schmitt, 2010). In this regard, as Yoon (2013) suggests reading ebooks may also help EFL learners develop and expand their vocabulary knowledge. Furthermore, the literature suggests that explicit instruction can (or even should) be incorporated in dealing with new vocabulary (see Barcroft, 2009; Ciftci \& Uster, 2009; Lee, 2003; Shintani, 2013). Thus, the researchers recommend that language teachers devote some time (as a part of an intensive, systematic recycling) to highlight the pragmatic aspects of such troublesome words.

In addition, language and culture are closely related to each other, and language is the vehicle of cultural manifestation (Zhao, 2004). Therefore, EFL learners' failure to recognize the cultural connotations of L2 words could easily cause serious misunderstanding in their communication with native speakers (Jalali \& Tamimi-Sa'd, 2014). Hence, an integrated approach combining culture and language is strongly recommended. In this regard, cultural portfolios can be an effective tool to teach EFL learners about the target language and culture (Byon, 2007). Using cultural portfolios, learners have the opportunity to get involved in cultural topics, based on their personal interests, to gain both language skills and cultural understanding.

Finally, although, as mentioned in the discussion, L1 translation is a natural part of L2 lexical processing and a popular strategy for EFL learners (see for example, Calis \& Dikilitas, 2012; Celik \& Toptas, 2010; 
The effects of L1 lexicalization and cultural loadedness on incidental vocabulary acquisition and retention

Hummel, 2010; Tagashira, Kida, \& Hoshino, 2010; Yau, 2010), due to the formal and semantic mismatch between NL and CL words on the one hand and their L1 equivalents on the other hand, language teachers had better prepare a learning context where their learners can acquire these words directly from the surrounding spoken or written context (see Borovsky, Kutas, \& Elman, 2010; Laufer \& Paribakht, 1998;Webb, 2008) . The second choice at hand is, of course, form-focused instruction within a communicative perspective of language teaching and learning (see Barcroft, 2009; Schmitt, 2008; Shintani, 2013; Tang \& Nesi, 2003 on explicit vocabulary instruction).

This study had its own limitations. Firstly, it was limited in the number of the TWs and the participants used. Certainly, a larger number of participants from different age groups and proficiency levels could expand its generalizability to other EFL contexts. Moreover, using qualitative measures such as think-aloud protocols could open a window to the learners' lexical processing and improve the validity of the findings; this study looked into the topic from a bird's eye view, seeking for the patterns of difference at macro level. Further studies may address the same issue from an explicit instruction perspective, employing a larger sample of participants and TWs. In addition, it would be interesting to see if the same results would be obtained and retained in longer periods of time. Thus, a longitudinal study investigating the acquisition of NL and/or CL words using both quantitative and qualitative measures of vocabulary knowledge can be an appropriate follow-up to this study.

Acknowledgements: The researchers would like to thank the participants of the study and the volunteers who kindly helped in the collection and validation of the TWs.

\section{References:}

Ang, I. (2014). Rethinking the role of context and definition in second language vocabulary acquisition (SLVA): An assimilation via a cognitive model of concept formation. International Journal of Research Studies in Language Learning, 3(1), 33-50. http://dx.doi.org/10.5861/ijrsll.2013.515

Barcroft, J. (2002). Semantic and structural elaboration in L2 lexical acquisition. Language Learning, 52(2), 323-363. http://dx.doi.org/10.1111/0023-8333.00186

Barcroft, J. (2009). Strategies and performance in intentional L2 vocabulary learning. Language Awareness,

\section{8(1), 74-89. http://dx.doi.org/10.1080/09658410802557535}

Brent, M. R., \& Siskind, J. M. (2001). The role of exposure to isolated words in early vocabulary development. Cognition, 81, 33-44. http://dx.doi.org/10.1016/S0010-0277(01)00122-6

Borovsky, A., Kutas, M., \& Elman, J. (2010). Learning to use words: Event-related potentials index single-shot contextual word learning. Cognition, 116, 289-296. http://dx.doi.org/10.1016/j.cognition.2010.05.004

Brown, R., Waring, R., \& Donkaewbua, S. (2008). Incidental vocabulary acquisition from reading, reading-while-listening, and listening to stories. Reading in a Foreign Language, 20(2), 136-163.

Byon, A. S. (2007). The use of cultural portfolio project in Korean culture classroom: Evaluating stereotypes and enhancing cross-cultural awareness. Language, Culture, and Curriculum, 20, 1-19. http://dx.doi.org/10.2167/lcc323.0

Calis, E., \& Dikilitas, K. (2012). The use of translation in EFL classes as L2 learning practice. Procedia: Social and Behavioral Sciences, 46, 5079-5084.

Celik, S., \& Toptas, V. (2010). Vocabulary learning strategy use of Turkish EFL learners. Procedia: Social and Behavioral Sciences, 3, 62-71

Chen, C., \& Truscott, J. (2010).The effects of repetition and L1 lexicalization on incidental vocabulary acquisition. Applied Linguistics, 31(5), 693-713. http://dx.doi.org/10.1093/applin/amq031

Choo, L. B., Lin, D. T. A., \& Pandian, A. (2012). Language learning approaches: A review of research on explicit and implicit learning in vocabulary acquisition. Procedia: Social and Behavioral Sciences, 55, 852-860.

Ciftci, H., \& Uster, S. (2009). A comparative analysis of teaching vocabulary in context and by definition. Procedia: Social and Behavioral Sciences, 1, 1568-1572. 
Heidari-Shahreza, M. A., Moinzadeh, A., \& Barati, H.

Corrigan, R. (2007). An experimental analysis of the affective dimensions of deep vocabulary knowledge used in inferring the meaning of words in context. Applied Linguistics, 28(2), 211-240. http://dx.doi.org/10.1093/applin/amm009

Crossley, S. A., Salsbury, T., \& McNamara, D. S. (2011). Predicting the proficiency level of language learners using lexical indices. Language Testing, 29(2), 243-263. http://dx.doi.org/10.1177/0265532211419331

Day, R. R., \& Bamford, J. (2002). Top ten principles for teaching extensive reading. Reading in a Foreign Language 14(2), 136-141.

Erten, I. H., \& Tekin, M. (2008). Effects on vocabulary acquisition of presenting new words in semantic sets versus semantically unrelated sets. System, 36, 407-422. http://dx.doi.org/10.1016/j.system.2008.02.005

Ghafar-Samar, R., \& Dehqan, M. (2013). Sociocultural theory and reading comprehension: The scaffolding of readers in an EFL context. International Journal of Research Studies in Language Learning, 2(3), 67-80. http://dx.doi.org/10.5861/ijrsll.2012.183

Golaghaei, N., \& Sadighi, F. (2013). L1 glossing and lexical inferencing: evaluation of the overarching issue of L1 lexicalization. The Journal of Teaching Language Skills, 4(4), 1-24.

Hairrell, A., Rupley, W., \& Simmons, D. (2011). The State of vocabulary research. Literacy Research and Instruction, 50(4), 253-271. http://dx.doi.org/10.1080/19388071.2010.514036

Hall, C. J. (2002). The automatic cognate form assumption: Evidence for the Parasitic Model of vocabulary development. International Review of Applied Linguistics, 40, 69-87. http://dx.doi.org/10.1515/iral.2002.008

Hamada, M. (2009). Development of L2 word-meaning inference while reading. System, 37, 447-460. http://dx.doi.org/10.1016/j.system.2009.03.003

Heidari-Shahreza, M. A., \& Tavakoli, M. (2012). The effects of repetition and L1 lexicalization on incidental vocabulary acquisition by Iranian EFL Learners. The Language Learning Journal, 40, 1-16. http://dx.doi.org/10.1080/09571736.2012.708051

Hellman, A. B. (2011). Vocabulary size and depth of word knowledge in adult-onset second language acquisition. International Journal of Applied Linguistics, 21(2), 162-182. http://dx.doi.org/10.1111/j.1473-4192.2010.00265.x

Hummel, K. M. (2010). Translation and short-term L2 vocabulary retention: Hindrance or help? Language Teaching Research, 14(1), 61-74. http://dx.doi.org/10.1177/1362168809346497

Jalali, S., \& Tamimi-Sa'd, S. H. (2014). Culture in ELT: A probe into Iranian EFL teachers' perspectives. International Journal of Research Studies in Language Learning, 3(3), 15-28 http://dx.doi.org/10.5861/ijrs1l.2013.599

Jiang, N. (2004). Semantic transfer and its implications for vocabulary teaching in a second language. The Modern Language Journal, 88, 416-432. http://dx.doi.org/10.1111/j.0026-7902.2004.00238.x

Karami, M., \& Salahshoor, F. (2014). The relative significance of lexical richness and syntactic complexity as predictors of academic reading performance. International Journal of Research Studies in Language Learning, 3(2), 17-28 http://dx.doi.org/10.5861/ijrsll.2013.477

Keiffer, M. J., \& Lesaux, N. K. (2012). Knowledge of words, knowledge about words: Dimensions of vocabulary in first and second language learners in sixth grade. Reading and Writing 25, 347-373. http://dx.doi.org/10.1007/s11145-010-9272-9

Khatib, M., \& Nourzadeh, S. (2012). Incidental L2 vocabulary acquisition through reading comprehension: Issues, agreements and controversies. Journal of Academic and Applied Studies, 2(2), 1-11.

Kim, Y. J. (2011). The role of task-induced involvement and learner proficiency in L2 vocabulary acquisition. Language Learning, 61(1), 100-140. http://dx.doi.org/10.1111/j.1467-9922.2011.00644.x

Kirn E., \& Hartmann, P. (2002). Interactions 1: Reading. New York: McGraw-Hill/Contemporary.

Kupelian, M. (2001). Korean EFL Students' Acquisition of Culturally Loaded Words. The Korea TESOL Journal, 4(1), 17-36.

Larrañaga, P., Treffers-Daller, J., Tidball, F., \& Ortega, M. G. (2012). L1 transfer in the acquisition of manner and path in Spanish by native speakers of English. International Journal of Bilingualism, 16(1), 117-138. http://dx.doi.org/10.1177/1367006911405577 
The effects of L1 lexicalization and cultural loadedness on incidental vocabulary acquisition and retention

Laufer, B., \& Paribakht, T. S. (1998). The relationship between passive and active vocabularies: Effects of language learning context. Language Learning, 48(3), 365-391. http://dx.doi.org/10.1111/0023-8333.00046

Laufer, B., Elder, C., Hill, K., \& Congdon, P. (2004). Size and strength: Do we need both to measure vocabulary knowledge? Language Testing, 21(2), 202-226. http://dx.doi.org/10.1191/0265532204lt277oa

Laufer, B., \& Goldstein, Z. (2004). Testing vocabulary knowledge: Size, strength and computer adaptiveness. Language Learning, 54(3), 399-436. http://dx.doi.org/10.1111/j.0023-8333.2004.00260.x

Lee, S. H. (2003). ESL learners' vocabulary use in writing and the effects of explicit vocabulary instruction. System, 31, 537-561. http://dx.doi.org/10.1016/j.system.2003.02.004

Liu, D., \& Zhong, S. (1999). Acquisition of culturally loaded words in EFL. Foreign Language Annals, 32(2), 177-187. http://dx.doi.org/10.1111/j.1944-9720.1999.tb02390.x

Marzban, A., \& Hadipour, R. (2012). Depth versus breadth of vocabulary knowledge: assessing their roles in Iranian intermediate EFL students' lexical inferencing success through reading. Procedia: Social and Behavioral Sciences, 46, 5296-5300.

Mehrpour, S., \& Rahimi, M. (2010). The impact of general and specific vocabulary knowledge on reading and listening comprehension: A case of Iranian EFL learners. System, 38, 292-300. http://dx.doi.org/10.1016/j.system.2010.01.004

Min, H-T. (2008). EFL vocabulary acquisition and retention: reading plus vocabulary enhancement activities and narrow reading. Language Learning, 58(1), 73-115. http://dx.doi.org/10.1111/j.1467-9922.2007.00435.x

Nation, I. S. P. (1990). Teaching and Learning Vocabulary. New York, NY: Heinle and Heinle Publishers.

Nation, I. S. P. (2001). Learning Vocabulary in another Language. Cambridge: Cambridge University Press. http://dx.doi.org/10.1017/CBO9781139524759

Nation, I. S. P. (2013). Vocabulary acquisition in second language acquisition. In C. A. Chapelle (Ed.), The Encyclopedia of Applied Linguistics. Oxford: Blackwell Publishing Ltd.

Paribakht, T. S. (2005). The influence of first language lexicalization on second language lexical inferencing: A study of Farsi-speaking learners of English as a foreign language. Language Learning, 55(4), 701-748. http://dx.doi.org/10.1111/j.0023-8333.2005.00321.x

Pellicer-Sanchez, A., \& Schmitt, N. (2010). Incidental vocabulary acquisition from an authentic novel: Do Things Fall Apart? Reading in a Foreign Language, 22(1), 31-55.

Pulido, D. (2004). The relationship between text comprehension and second language incidental vocabulary acquisition: A matter of topic familiarity? Language Learning, 54, 469-53. http://dx.doi.org/10.1111/j.0023-8333.2004.00263.x

Qi, S. (1992). A Comparative Case Study of Prior Cultural Knowledge in English-Second-Language Lexical Meaning-Making (Master's thesis). Available from Eric database. (ED351873).

Qian, D. D., \& Schedl, M. (2004). Evaluation of an in-depth vocabulary knowledge measure for assessing reading performance. Language Testing, 21(1), 28-52. http://dx.doi.org/10.1191/02655322041t273oa

Rott, S. (2013). Incidental vocabulary acquisition. In C. A. Chapelle (Ed.), The Encyclopedia of Applied Linguistics. Oxford: Blackwell Publishing Ltd.

Schmitt, N. (2008). Review article: Instructed second language vocabulary learning. Language Teaching Research, 12(3), 329-363. http://dx.doi.org/10.1177/1362168808089921

Schmitt, N., Schmitt, D., \& Clapham, C. (2001). Developing and exploring the behavior of two new versions of the Vocabulary Levels Test. Language Testing, 18(1), 55-88.

Shintani, N. (2013). The effect of focus on form and focus on forms instruction on the acquisition of productive knowledge of L2 vocabulary by young beginning-Level learners. TESOL Quarterly, 47(1), 36-62. http://dx.doi.org/10.1002/tesq.54

Tagashira, K., Kida, S., \& Hoshino, Y. (2010). Hot or gelid? The influence of L1 translation familiarity on the interference effects in foreign language vocabulary learning. System, 38, 412-421. http://dx.doi.org/10.1016/j.system.2010.03.015

Tang, E., \& Nesi, H. (2003). Teaching vocabulary in two Chinese classrooms: schoolchildren's exposure to English words in Hong Kong and Guangzhou. Language Teaching Research, 7(1), 65-97. 
Heidari-Shahreza, M. A., Moinzadeh, A., \& Barati, H.

http://dx.doi.org/10.1191/13621688031r113oa

Webb, S. (2007). The effects of repetition on vocabulary knowledge. Applied Linguistics, 28(1), 46-65. http://dx.doi.org/10.1093/applin/aml048

Webb, S. (2008). The effects of context on incidental vocabulary learning. Reading in a Foreign Language, 20(2), 232-245.

Webb, S. (2009). The Effects of Receptive and Productive Learning of Word Pairs on vocabulary knowledge. RELC Journal, 40(3), 360-376. http://dx.doi.org/10.1177/0033688209343854

Webb, S. (2013). Depth of vocabulary knowledge. In C. A. Chapelle (Ed.), The Encyclopedia of Applied Linguistics. Oxford: Blackwell Publishing Ltd.

Yau, J. C. (2010). Roles of mental translation in first and foreign language reading. International Journal of Bilingualism, 15(4), 373-387. http://dx.doi.org/10.1177/1367006910380038

Yoon, T. (2013). Beyond the traditional reading class: The application of an e-Book in EFL English classroom. International Journal of Research Studies in Language Learning, 2(1), 17-26 http://dx.doi.org/10.5861/ijrsll.2012.93

Zhao, J. (2004). ESL students' awareness of cultural connotations of words. Arizona Working Papers in SLAT, 11, $37-55$.

\section{Appendix}

An example of the main reading passages

$>\quad$ Bolded words represent lexicalized (L) words;

$>\quad$ Italic words represent non-lexicalized (NL) words;

$>\quad$ Underlined words represent culturally-loaded (CL) words;

> Strikethrough-words represent culturally-neutral (CN) words.

He was a stubborn person and it explained why he had few friends. What was more, he was too abstinent; he had never gambled or even as an young adult, dated a girl. Yet, strangely enough, there was some rumor in the neighborhood that he used to smuggle cocaine and eloped with his girlfriend once to Las Vegas. As a retired artist, he most often sat in the lounge of Hotel Mirage, 3 minutes away from his home, to eat his brunch and giggle at the travelers who came and went. Some actually thought he was idiot. But indeed, he was just alone and tired of his whereabouts. Few people liked him. While he was real annoyance to the hotel staff, Mr. Hathaway, the hotel manager was kind to him because old George once had presented his masterpiece to the hotel. It had been hung on the wall at Mr. Hathaway's office for years. There was a joke among hotel staff about the artist. They said you could not flee from two persons: an undertaker and old George. Anyway, he suited the smoggy city of Chicago well; where your best friend is your pet, usually a dog and where you meet your neighbors accidentally at the junction of a road or on a wholesale market. 